\title{
Relationship between Maturation of the Skin and Electrical Skin Resistance
}

\author{
KAZUHIKO MURAMATSU, SHINICHI HIROSE, KOU YUKITAKE, HIROKO OGATA, \\ AKIHISA MITSUDOME, AND TEIICHI ODA
}

Department of Pediatrics, Fukuoka University, Fukuoka, Japan

\begin{abstract}
In 16 newborn infants (24 6-39 wk 6 days 830-3390g) at ages between 0-70 days, the skin's electrical resistance was measured. Over 30 min we sequentially measured direct current resistance between two skin electrodes placed on the abdomen. The resistance was found to be low in very premature infants with gestation ages of 30 wk or less. It increased with both gestational age and postnatal age. In these infants, the resistance measured at 30 min during the first 4 days of life $(3.4 \pm 0.3 \mathrm{~K} \Omega$; mean \pm SD) was significantly less than that found at 20-70 days $(8.8 \pm 1.2 \mathrm{k} \Omega ; p<0.001)$. The relationship between the infant's electrical skin resistance $(y)$ and the gestational age $(x)$ best fit the exponential formula: $y=7.42 \cdot e^{0.24 x}$. $10^{-4}+2.74$. This formula shows that the electrical skin resistance increases exponentially during the last trimester. The measurement of electrical skin resistance is a quantitative method that can be utilized to evaluate the gestational age of infants. (Pediatr Res 21: 21-24, 1986)
\end{abstract}

It has been demonstrated that electrical skin resistance is exclusively located within the stratum corneum $(1,2)$, and the resistance is directly related to the thickness of the stratum corneum $(3,4)$. The skin of premature infants is thin, gelatinous, edematous, translucent (5), and has little capacity to produce sweat (6). These skin properties of newborn infants are important characteristics related to gestational age. Although the assessment of these characteristics is simple, they are unfortunately qualitative in nature. The effect of these premature skin characteristics on the electrical resistance of the skin may change with advancing gestational age and therefore skin resistance may provide a quantitative measure of skin maturity. This study was designed to evaluate the relationship between the electrical skin resistance and the skin maturity of newborn infants.

\section{MATERIALS AND MFTHODS}

All infants studied were born in the Fukuoka University Hospital and were selected on the basis of maternal menstrual history. For analysis we used infants of mothers who were certain of the date of her last menstrual period, had regular 28 day $( \pm 2$ days) cycles, and had no bleeding subsequent to their last menstrual period. Maternal parity and method of delivery were not considered in the selection. During the first 2 days of life we used the postnatal scoring system as described by Dubowitz et al. (7) and/or Ballard et al. (8) to determine the gestational age. When a discrepancy between the gestational age as calculated by menstrual history and that as calculated from the scoring system was $3 \mathrm{wk}$ or more, the infants were excluded from the study. Informed

Received March 11, 1986: accepted August 7, 1986.

Reprint requests Kazuhiko Muramatsu, M.D., Department of Pediatrics, Fukuoka Tokushukai Hospital, 960-2 Suku. Kasuga City, Fukuoka, 816. Japan. consent was obtained from the parents. In the study of the skin, investigators have attempted to utilize measured electrical skin resistance both to direct current and alternating current. We arbitrarily chose to measure resistance to direct current. The electrodes were stainless steel disks $1.5 \mathrm{~cm}$ in diameter. Two electrodes were placed on the skin at a fixed distance of $3 \mathrm{~cm}$ between their centers. The electrodes were applied with rubber straps as commonly used in electrocardiographs which allowed a nearly constant pressure with each application. The electrical connection to the skin was made using electrocardigram electrode jelly (Keratin cream, Fukuda Co., Tokyo, Japan). The electrodes were always applied to the surface of the abdomen midway between the xiphisternum and the umbilicus.

Prior to the application of the electrodes, the skin surface was cleansed with a wet towel and then with ethanol to remove the vernix caseosa and other lipid materials. Immediately after the electrodes were positioned on the skin a constant voltage of 0.4 $\mathrm{V}$ was delivered between the two electrodes. The electrical current through the skin ranged between 5 and 150 microamperes. An ohm-meter (Hi-calc tester, Hioki Co., Tokyo, Japan) was used to measure skin resistance to direct current. Two resistors of $3 \mathrm{k} \Omega$ and $50 \mathrm{k} \Omega$ (usual fixed resistors for electrical circuits) were used in the calibration of the ohm-meter. The changes in resistance between the two electrodes were recorded sequentially at 2-min intervals for $10 \mathrm{~min}$, and then at $5 \mathrm{~min}$ intervals for $20 \mathrm{~min}$. The timer was started when the electrode jelly was applied to the skin. It was observed that visible sweat gland activity decreased electrical skin resistance (9). To avoid this effect on resistance by perspiration, the skin temperatures were held at $36.5-37.0^{\circ} \mathrm{C}$. Infants with body weights of $2000 \mathrm{~g}$ or less were nursed in servocontrolled incubators with environmental temperatures maintained at 31.4 to $36.0^{\circ} \mathrm{C}$. Infants with body weights of 2001 $\mathrm{g}$ or more were nursed in cots with light dressing. Room temperatures were held between $20-25^{\circ} \mathrm{C}$. If the skin temperatures were more than $37.1^{\circ} \mathrm{C}$, and/or if perspiration was visibly evident, the study was postponed.

Every infant was first studied during the first 4 days of life and infants with gestational ages of $32 \mathrm{wk}$ or less were subsequently studied at 1- to 2-wk intervals. The results of the measurements of electrical skin resistance were subjected to statistical analysis using the two tailed Student's $t$ test. All data are expressed as mean values $\pm 1 \mathrm{SD}$.

\section{RESULTS}

We studied the electrical characteristics of the skin of 16 newborn infants ( 24 to 39 wk 6 days; 830 to $3390 \mathrm{~g}$ ). The results of the investigation are summarized in Table 1. On initial contact between the electrodes and the skin the resistance was high but fell rapidly to reach a nearly steady value within $30 \mathrm{~min}$ of contact (Fig. 1). In the first 4 days of life the electrical resistance of very premature infants with gestational ages of $30 \mathrm{wk}$ or less $(27.9 \pm 2.1 \mathrm{wk})$ were low throughout the 30 -min study period; 
Table 1. Electrical resistance of the skin in newborn infants

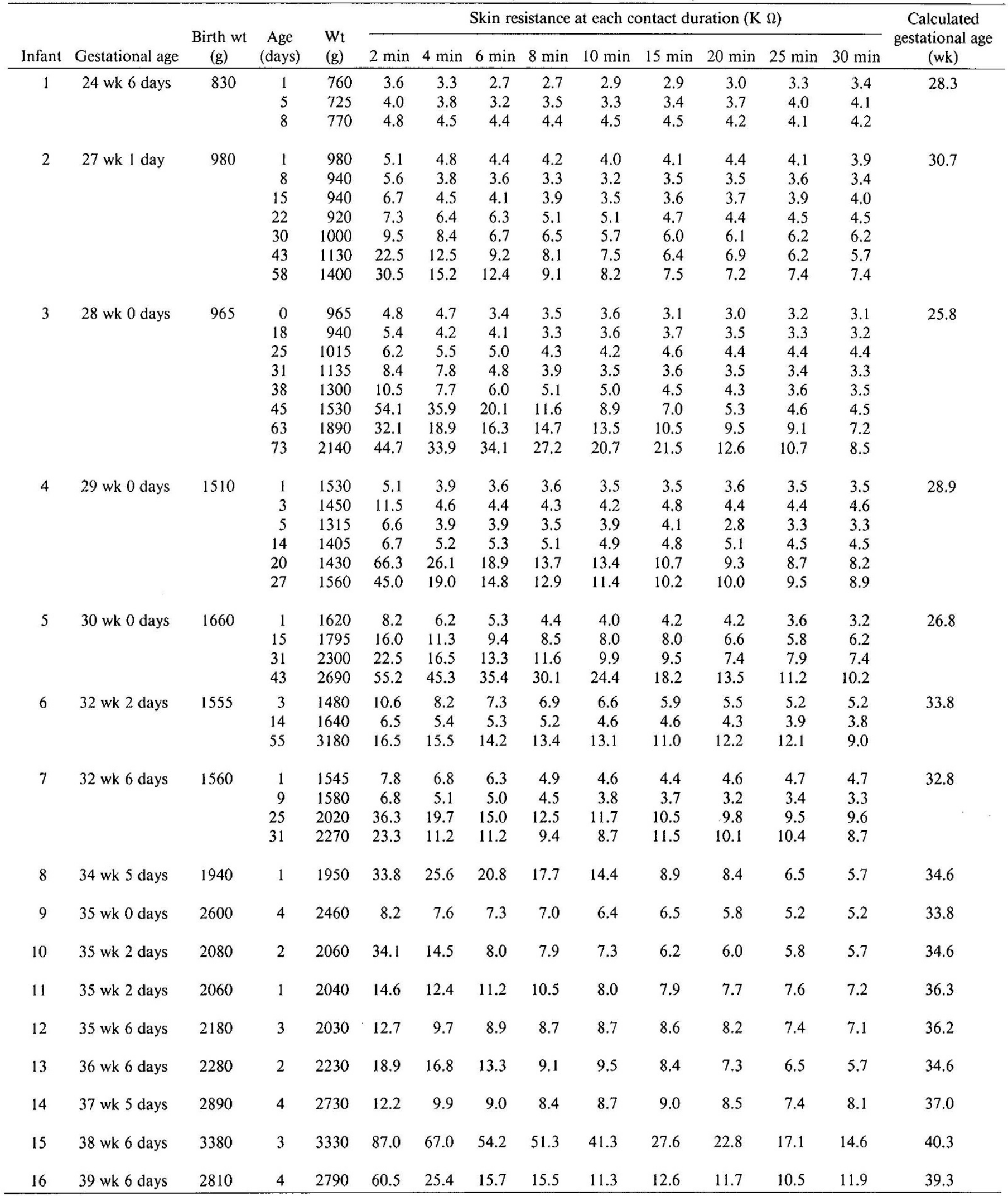

however, at older postnatal ages (20-70 days) the resistances were significantly higher during the first $10 \mathrm{~min}$ and remained so at the $30 \mathrm{~min}$ measurement (Fig. 1). In general, the pattern of resistance decline for these premature infants at older postnatal ages was similar to the pattern for infants born at gestational age of 34 wk or more at 0-4 days of life (Table 1).

There appears to be an exponential relationship between gestational ages and skin resistances (Fig. 2). The values for skin 
resistance in Figure 2 were obtained during the first 4 days of life and were those measured at $30-$ min intervals in each study. The most representative regression formula for gestational age $(x)$ against the electrical skin resistance $(\mathrm{y})$ is: $\mathrm{y}=7.42 \cdot e^{0.24 x} \cdot 10^{-4}+$ $2.74(r=0.92)$.

This formula shows that the electrical skin resistance increases exponentially during the last trimester. Gestational age $(\mathrm{x})$ can be expressed as follows: $x=\log _{c}(y-2.74)+7.21 / 0.24$. The calculated gestational ages are shown in Table 1. The mean discrepancy between gestational ages as calculated by menstrual history and those as calculated from the equation is $1.36 \pm 1.24$ wk.

The relationship between the skin resistance and the postnatal age is shown in Figure 3. The values for skin resistance in figure 3 were those obtained at the 30 -min time point in each study. The skin resistance increased gradually in infants with the gestational ages of $28 \mathrm{wk}$ or less and birth weights of $1000 \mathrm{~g}$ or less, and increased rapidly in infants with gestational ages of $29-32$

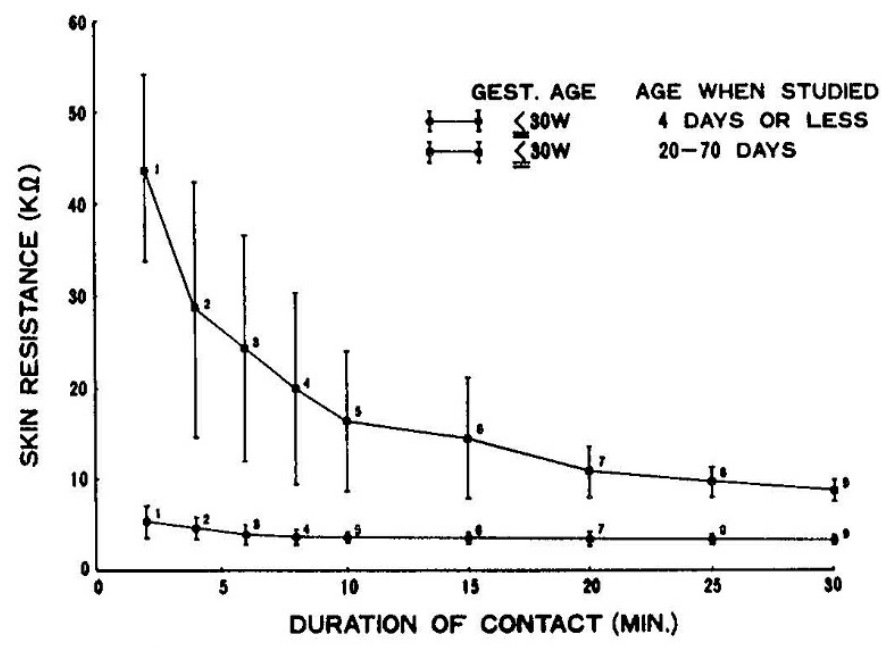

Fig. 1. The electrical skin resistance of newborn infants with gestational ages of $30 \mathrm{wk}$ or less at 0-4 and 20-70 days of age. Closed circles, $0-4$ days, $n=5$; closed squares, 20-70 days, $n=4$; values $=$ means \pm 1 SD. Comparisons in 1, 7, 8, 9 and 2,3,4,5,6 are $p<0.001$ and $p<0.01$, respectively. Note that the resistance is significantly higher in the later postnatal period. The resistance decreases rapidly with older postnatal ages.

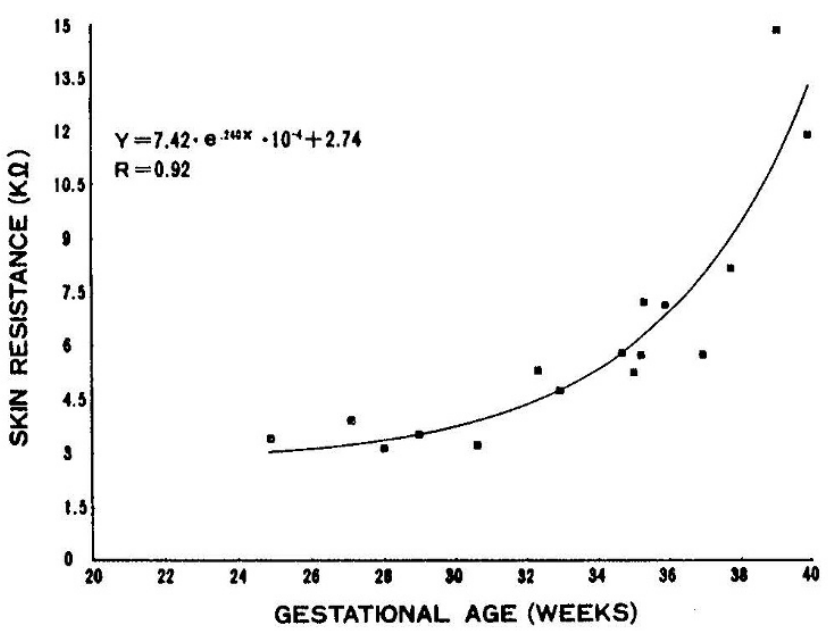

Fig. 2. Relationship between the gestational age and the electrical resistance of the skin in newborn infants. Note that the electrical resistances increase rapidly after about $32 \mathrm{wk}$ of gestation.

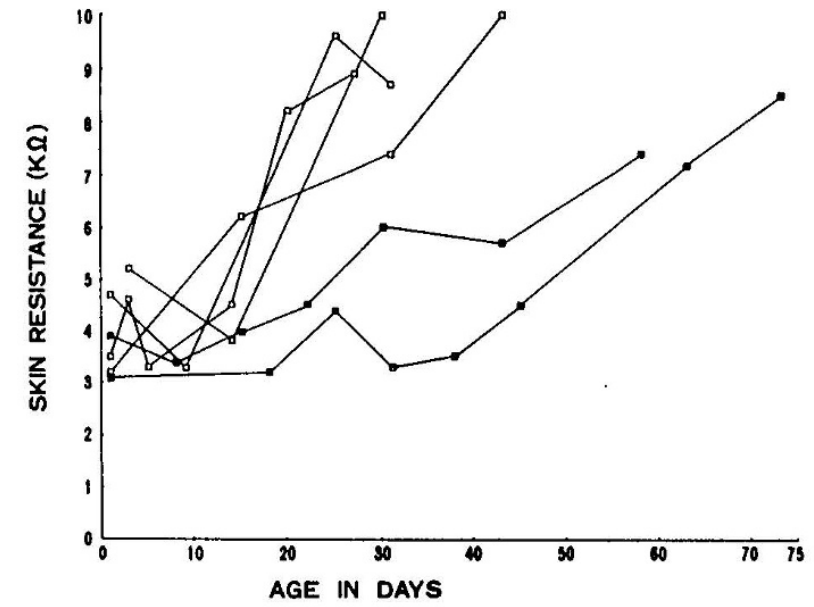

Fig. 3. Relationship between the postnatal age and the skin resistance measured for $30 \mathrm{~min}$ in infants born at gestational ages of $32 \mathrm{wk}$ or less. Open squares, gestational age 29-32 wk, birth weight 1510-1660 g. Closed squares, gestational age $27-28$ wk, birth weight $965-980 \mathrm{~g}$.

wk and birth weights of $1500-1660 \mathrm{~g}$. Although there were no significant differences in electrical skin resistance measured during the first 10 days of life between the two groups, the developmental patterns were different.

\section{DISCUSSION}

We have shown that the skin resistance of the newborn infant is related to the gestational age of the infant. In addition the resistance also changes with the postnatal age of the infant.

The skin of premature infants is very thin and gelatinous and becomes thicker and more firm with advancing gestational age. Physical skin characteristics such as texture, color, opacity, edema, and planter creases provide valuable information in the assessment of gestational age. Farr et al. (10) reviewed a number of external characteristics which they found to be useful in the assessment of gestational age and developed a system of scoring each criterion. In a series of 272 infants, the characteristics they studied were skin texture, skin color, skin opacity, planter skin creases, skin edema, lanugo, ear form, ear firmness, genitalia development, breast size, and nipple formation. The assessment score using these characteristics was closely correlated with the gestational age for boys $(r=0.75)$ and for girls $(r=0.77)$. Five of the 11 criteria adopted for this scoring system involve the skin. Although estimates of gestational age are heavily dependent on the maturation of the skin, the changes of skin characteristics determined by inspection or palpation are merely qualitative assessment. These methods are useful in clinical practice, but they lack precision and accuracy. The method described herein is a quantitative analysis of an electrical characteristic of the skin. Investigators $(2,3)$ have demonstrated that the electrical skin resistance/impedance is exclusively located within the stratum corneum. Lawler $e$ al. (4) reported that by removing the stratum corneum using cellulose tape stripping the skin lost its resistance. They found that there was a proportional decrease in skin resistance during the gradual removal of the stratum corneum. Gerstner and Gerbstadt (3) demonstrated that the skin resistance is related to the thickness of the keratin layer, i.e. a thin keratin layer had a lower resistance than in those areas in which the keratin layer was thick. We found that skin resistance increases in accordance with the advancing gestational age of newborn infants (Fig. 2). After birth the skin resistance increased gradually during the subsequent weeks in very premature infants and rapidly in the more mature infants (Fig. 3). These findings indicate that the skin resistance correlates with infant maturity. 
Tregear (11) found that skin impedance was much less with wet electrodes than with dry electrodes. He concluded that the high impedance observed with dry electrodes could be due to a drying effect and poor conduction of the area under the electrodes; the low impedance observed with wet electrodes could be due to the moisture of the surrounding area. Grimnes (12) also demonstrated a rapid impedance reduction in the skin during a 10-min period using pregelled electrode. He concluded that this impedance reduction was due to electrolyte diffusion into the skin and thus increasing the effective electrode area. Our results also demonstrated the skin resistance decreased rapidly over the first $20 \mathrm{~min}$ when using wet electrodes. We postulated that the application of wet electrodes gradually increased the moisture and electrolyte content within the stratum corneum thus resulting in a decreased electrical resistance over the 30-min study period. In very premature infants during the first 4 days of life, the skin resistance was very low from the very beginning and did not show such a resistance reduction (Fig. 1). This finding is explained by the increased water content and increased permeability of the premature skin. Premature skin is so thin and permeable that insensible water loss can be a very real problem in very premature infants. Fanaroff et al. (13) reported that skin factors responsible to the large water loss in immature infants include a thinner epidermis, increased water content, and increased permeability of the skin. They observed disproportionally large water losses in infants with birth weights less than $1250 \mathrm{~g}$ and gestational ages less than 230 days. This finding agrees with our results which suggest that skin maturation does not make much progress until about $32 \mathrm{wk}$ of gestation.

Perspiration decreases the electrical resistance of the skin $(9$, 14). Thomas and Korr (9) reported that secreting sweat gland lowers resistance in two ways; by serving as an added parallel high resistance in the electrical circuit and through the influence of moistening the skin. The influence of the sweat glands moistening the skin may be less distinct when using wet electrodes for long periods. The activity of sweat glands is poor in newborn infants $(6,15)$. In mature neonates to initiate the sweating response requires three times the thermal stimulus of that of an adult (6). In low birth weight infants, the sweating response to this thermal stimulus is much less developed than in mature infants (6). If no visible sweat gland activity is evident in newborn infant, the factor of added parallel high resistance in the electrical circuit is probably negligible.

Lawler et al. (4) states that utilizing direct current to measure electrical skin characteristic has the problem of establishing polarization currents within the body. The values of skin resist- ance as measured by skin electrodes include electrode polarization resistance, electrolyte resistance, and tissue resistance. Despite this our data obtained by utilizing direct current indicate that there is good correlation between the maturation of the skin and the direct current resistance of the skin in newborn infants.

As an estimate the skin's electrical resistance to direct current is useful as an index for skin maturation. We conclude that the measurement of skin resistance may provide a quantitative assessment of the gestational age of newborn infants. The discrepancy between gestational ages as calculated by menstrual history and those as calculated from our equation are small in premature infants especially in infants with gestational age $32 \mathrm{wk}$ or more (Table 1). A study of a larger population of infants will be necessary to define the accuracy and precision of this technique.

Acknowledgment. The authors thank Dr. Daniel T. Murai for preparation of the manuscript.

\section{REFERENCES}

1. Rosendal T 1943 Studies on the conducting properties of the human skin to direct current. Acta Physiol Scand 5:131-151

2. Yamamoto T, Yamamoto Y 1976 Electrical properties of the epidermal stratum corneum. Med Biol Eng Comput 14:151-158

3. Gerstner H, Gerbstadt H 1949 Der Wechselstrom widerstand der menschlichen Haut. Arch Physiol 252:111-119

4. Lawler JC, Michael JD, Griffith EC 1960 Electrical characteristics of the skin: The impedance of the surface sheath and deep tissues. J Invest Dermatol 34:301-308

5. Farr V, Mitchell RG, Neligan GA, Parkin JM 1966 The definition of some external characteristics used in the assessment of gestational age in the newborn infant. Dev Med Child Neurol 8:507-511

6. Behrendt H, Green M 1969 Drug-induced localized sweating in full-size and low-birth-weight neonates. Am J Dis Child 117:299-306

7. Dubowitz LMS, Dubowitz V, Goldberg C 1970 Clinical assessment of gestational age in the newborn infant. J Pediatr 77;1-10

8. Ballard JL, Novak KK, Driver M 1979 A simplified score for assessment of fetal maturation of newly born infants. J Pediatr 95:769-774

9. Thomas DE, Korr IM 1957 Relationship between sweat gland activity and electrical resistance of the skin. J Appl Physiol 10:505-510

10. Farr V, Kerridge DF, Mitchell RG 1966 The value of some external characteristics in the assessment of gestational age at birth. Dev Med Child Neurol 8:657-660

11. Tregear RT 1965 Interpretation of skin impedance measurements. Nature 205:600-601

12. Grimnes $\mathrm{S} 1983$ Impedance measurement of individual skin surface electrodes. Med Biol Eng Comput 21:750-755

13. Fanaroff AA, Wald M, Gruber HS, Klaus MH 1972 Insensible water loss in low birth weight infants. Pediatrics 50:236-245

14. Wagner HN 1952 Electrical skin resistance studies in two persons with congenital absence of sweat glands. Arch Derm Syphilol 65:543-548

15. Green M, Behrendt H 1973 Sweating responses of neonates to local thermal stimulation. Am J Dis Child 125:20-25 